\title{
Siete nuevos registros de Arctiini (Lepidoptera: Erebidae: Arctiinae) para Perú
}

\author{
Seven new records Arctiini (Lepidoptera: Erebidae: Arctiinae) to Peru
}

Juan Grados ${ }^{1}$, Carlos Espinoza², Juan José Ramírez ${ }^{3}$ y Pedro Centeno ${ }^{4}$

\begin{abstract}
1 Departamento de Entomología, Museo de Historia Natural, Universidad Nacional Mayor de San Marcos, Apartado 14-0434, Lima 14, Perú.

2 Universidad Nacional San Agustín, Arequipa, Perú.

3 Universidad Nacional de la Amazonía Peruana, Iquitos, Perú.

4 Botanical Research Institute of Texas, 500 E. 4th St., Fort Worth, TX 76102.

Email Juan Grados: gradosjuan@hotmail.com

Email Carlos Espinoza: cespinoza16@gmail.com Email Juan José Ramírez:macrodontia@hotmail.com
\end{abstract}

Citación:

Grados J., C. Espinoza, J.J. Ramírez \& P. Centeno. 2013 Siete nuevos registros de Arctiini (Arctiinae: Erebidae: Siete nuevos registros de Arctiini (Arctiinae: Erebidae:
Lepidoptera) para Perú. Rev. peru. biol. 20(2): 171- 176 (Diciembre 2013)

\section{Resumen}

Se presenta siete nuevos registros de Arctiini (Erebidae: Lepidoptera) para Perú. Algunas de las especies son raras en colecciones. Cada nuevo reporte pertenece a géneros diferentes, proporcionando para cada género las especies que ocurren en el Perú, basado en colecciones y las fuentes bibliográficas de las descripciones originales. Se da a conocer un nuevo sinónimo para Agyrtiola niepelti Gaede, 1926.

Palabras clave: Arctiini, Erebidae, Lepidoptera, nuevo sinónimo, Perú.

\section{Abstract}

Seven new Arctiini (Erebidae: Lepidoptera) are reported for Peru. Some of the species are rare among collections. Each new report belongs to different genera and the species given for each genus are those occurring in Peru, according to collections and literature references from their original descriptions. A new synonym is also reported for Agyrtiola niepelti Gaede, 1926.

Keywords: Arctiini, Erebidae, Lepidoptera, new synonym, Peru.

\section{Introducción}

El conocimiento de la riqueza de especies de los Arctiini (Erebidae) para la Región Neotropical ha sido registrada y revisada por Hampson (1901), Zerny (1912) y Watson y Goodger (1986).. Sin embargo, la existencia de sinónimos y confusiones taxonómicas han llevado a plantear que es un grupo donde se han sobreestimado los géneros y especies (Lamas y Grados 1996, Grados 2002, Cerda 2008). Por otro lado, es escasa la información acerca de la distribución geográfica de la mayoría de las especies de este grupo, donde muchas de las especies han sido descritas de ejemplares únicos, conociéndose solo su presencia en la localidad tipo.

Hasta la última década del siglo pasado, nuestro conocimiento de este grupo de lepidópteros provenían de varios colectores que recorrieron territorio peruano por diferentes circunstancias, entre los que encontramos a viajeros, exploradores, militares, artistas, antropólogos, capellanes, naturalistas y colectores profesionales. Los colectores que quizás más contribuyeron al conocimiento de los Arctiini del Perú fueron E. Böttger y G. R. Ockenden.

Emil Böttger (1863-1936) llega con sus padres y hermanos a Huancabamba (Pasco) en 1875, luego de dos momentos de colonización de los migrantes alemanes a Pozuzo (1857-1858; 1868-1869) (Sobrevilla 2001, Arona [1891]1971). Böttger colectó varios grupos de fauna, entre ellos los insectos en el valle de Huancabamba y otras localidades de lo que ahora forman parte del Parque Nacional Yanachaga-Chemillén. El material fue enviado probablemente a Hermann Rolle (1864-1929), comerciante alemán radicado en Berlín, de quien se conoce tenía material de Huancabamba y Pozuzo (Selva central), o quizás haya enviado material directamente a Lionel Walter Rothschild (1869-1937) del Tring Museum en Inglaterra (Jordan 1938, Lamas 1980). Todo el material de Böttger provenía de los alrededores de Huancabamba y no como en muchas publicaciones se menciona "Huancabamba, norte de Perú". Son muchas las

\section{Presentado: $\quad$ 06/04/2013}

Aceptado: $\quad 15 / 09 / 2013$ 


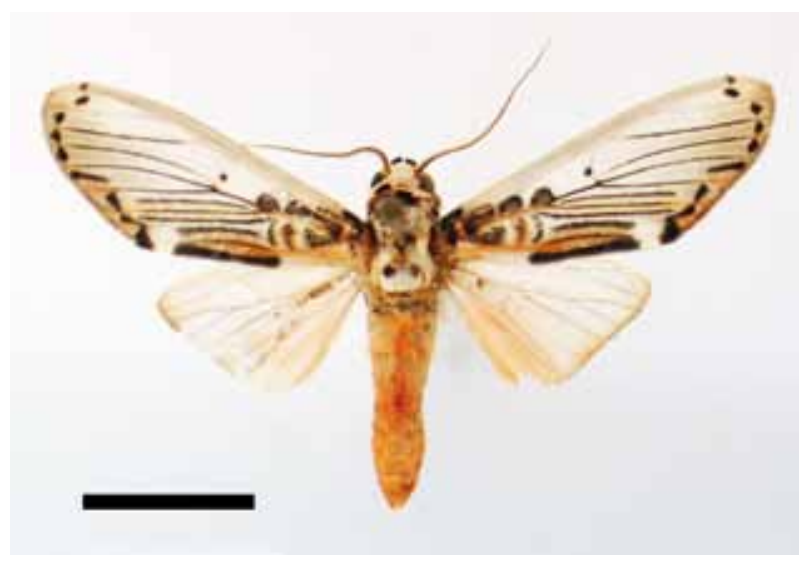

Figura 1. Aphyle onorei Toulgoët, 1988. Macho. Escala $1 \mathrm{~cm}$.

especies de Arctiini que fueron descritas con material colectado por Böttger, algunas de ellas endémicas y que actualmente estan depositadas en colecciones de diferentes museos.

En el sudeste de Perú, la labor de exploración biológica de George Richard Ockenden (Brighton, Inglaterra, 1868 - Puno, Perú, 1906) fue muy importante. Sus colectas las llevó a cabo en varios lugares como La Oroya, Santo Domingo, Oconeque, Agualani, Río Huacamayo, La Unión, Limbani, Chirimayo y Tinguri, todos cercanos al alto río Inambari, departamento de Puno. Ockenden recorrió por varios años estos parajes, aprovechando las vías de comunicación que tenía la Inca Mining Company y la Rubber Mining Company, desde la localidad de Tirapata (Puno) hasta Astillero, esta última en la boca del río Távara (Bailey 1906, Harter 1907, Bachmann 1918) casi límite entre los departamentos de Puno y Madre de Dios. El material colectado fue enviado a W.F.H. Rosenberg de Londres (Harter 1907). En la actualidad su material se encuentra en las colecciones del Museo Británico (BMNH) y en las del museo de la Universidad de Oxford (UMO).

Los nuevos registros presentados en este trabajo tienen relevancia porque en algunos casos amplían la distribución geográfica de especies raras y en otros porque proceden de zonas geográficas de difícil accesibilidad.

\section{Materiales}

Las colectas fueron llevadas a cabo entre los años 2004 y 2009, utilizando trampas de luz, utilizando lámparas de luz mixta (LLM) o de vapor de mercurio (LVM) y redes entomológicas para las especies de hábitos diurnos.

Las muestras fueron procesadas y depositadas en la colección del Museo de Historia Natural, Universidad Nacional Mayor de San Marcos, Lima (MUSM).

Los acrónimos empleados en este trabajo siguen a Heppner y Lamas (1982), habiendo sido revisadas las siguientes colecciones:

AMNH: American Museum of Natural History, New York.

MHNP: Muséum National d'Histoire Naturelle, Paris.

MUSM: Museo de Historia Natural, UNMSM, Lima.

USNM: United States National Museum, Smithsonian Institution, Washington.

ZMHB: Zoologisches Museum, Humboldt Universität, Berlín.

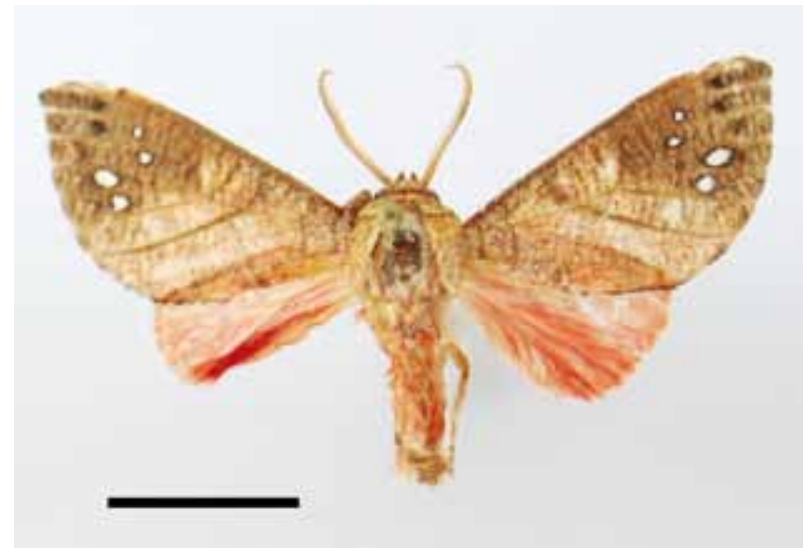

Figura 2. Zatrephes irrorata Rothschild, 1909. Macho. Escala $1 \mathrm{~cm}$.

Todos los nuevos registros pertenecen a diferentes géneros, por lo que también se proporciona una lista de las especies que ocurren en el Perú, indicándose entre paréntesis si existen ejemplares en la colección del Museo de Historia Natural de la Universidad Nacional Mayor de San Marcos, y en caso no esté representada en la colección, se señala donde se encuentra. Las especies que son reportadas en base a fuentes bibliográficas están señaladas con asterisco, adicionando el lugar exacto de colecta y el departamento si está especificado en la publicación.

Taxonomía

$\begin{array}{ll}\text { OrdEN: } & \text { LEPIDOPTERA } \\ \text { FAMILIA: } & \text { EREBIDAE } \\ \text { SuBFAMILIA: } & \text { ARCTIINAE } \\ \text { TrIBU: } & \text { ARCTIINI } \\ \text { SUBTRIBU: } & \text { PHAEGOPTERINA }\end{array}$

\section{Aphyle onorei Toulgoët, 1988}

(Fig. 1)

Especie muy llamativa por la coloración que presentan las alas. Fue descrita por Hervé de Toulgoët de un macho proveniente de Coca, Napo (Ecuador), colectada por G. Onore en el mes de enero (1988), a quien fue dedicada la especie.

Colectas llevadas a cabo en el departamento de Loreto han permitido registrar la especie más hacia el sur de su localidad tipo. El presente registro es el segundo macho que se conoce. La hembra es desconocida por ahora.

Material examinado: PERÚ: LORETO; $1 \hat{\jmath}$, Agua Blanca, 035'ㄱ / 73²8'W, 130m, 29.iv.2004 (J.J. Ramírez).

Otras especies del género registradas para Perú son: affinis Rothschild, 1909 (Aphyle)(Carabaya, Puno)* cuneata Hampson, 1905 (Aphyle) (MUSM) margaritaceus Walker, 1855 (Aphyle) (MUSM)

\section{Zatrephes irrorata Rothschild, 1909}

(Fig. 2)

Una especie aparentemente rara, habiendo sido descrita de un sólo ejemplar macho proveniente de Fonte Boa, Río Amazonas (Brasil), colectado en el mes de agosto (1906) por S.M. Klages. 


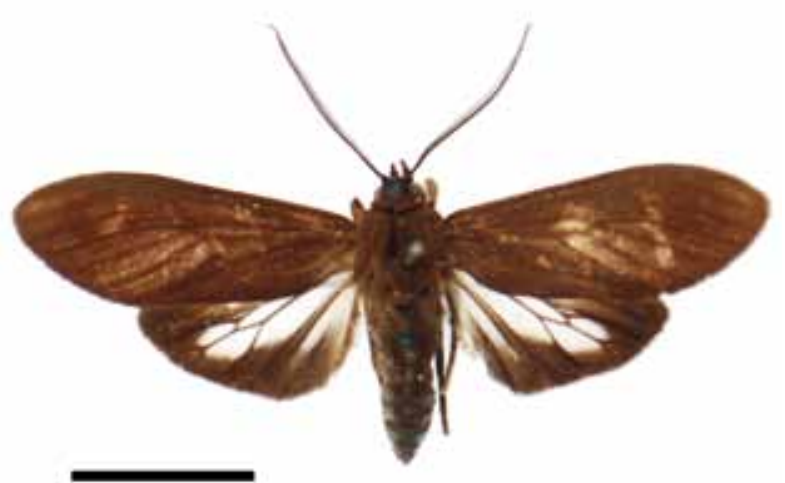

Figura 3. Aclytia ventralis (Guérin-Méneville, 1843). Macho. Escala $1 \mathrm{~cm}$.

Este primer registro para Perú proviene de una exploración biológica en la Cordillera Sierra del Divisor, frontera entre Perú y Brasil. Sólo se logró colectar un ejemplar macho, el segundo que se conoce de la especie en todo el mundo.

Material examinado: LORETO: $1 \hat{\jmath}, 37 \mathrm{~km}$ NE Monte Alegre, Río Tapiche, 06²3'01"S / 7404'35”W, $175 \mathrm{~m}$, 18-19.x.2008 (A. García).

Otras especies del género registradas para Perú son:

afenestrata Toulgoët. 1987 (Zatrephes)(MUSM)

bicolorata (Druce, 1906)(Apatelodes)(Santo Domingo, Puno)*

bilineata Rothschild, 1909 (Zatrephes)(Carabaya, Puno)*

brunnea Rothschild, 1909 (Zatrephes)(Carabaya, Puno)*

crocos (Cramer, 1773) (Phalaena) (MUSM)

cruciata Rothschild, 1909 (Zatrephes) (MUSM)

nitida (Cramer, 1780) (Phalaena) (MUSM)

ockendeni Rothschild, 1909 (Zatrephes)(La Oroya, Puno)*

propinqua (Rothschild, 1909) (Automolis)(Carabaya, Puno)*

pseudopraemolis (Rothschild, 1909) (Automolis) (MUSM)

trailli Butler, 1877 (Zatrephes) (MUSM)

trilineata peruviana Rothschild, 1910 (Zatrephes) (Carabaya y Santo Domingo, Puno)*

variegata (nr.) Rothschild, 1909 (Zatrephes) (MUSM)

SubTRIBU: CTENUCHINA

\section{Aclytia ventralis (Guérin-Méneville, 1843)}

(Fig. 3)

conspicua Druce, 1884:70 (Aclytia). Sinónimo.

lucania (Schaus, 1889): 89 (Glaucopis). Sinónimo.

La especie fue descrita de ejemplares colectados en México. De acuerdo a la bibliografía, la especie tiene como área de distribución México, Guatemala, Costa Rica y Panamá (Druce, 1884; Hampson, 1898).

El nuevo registro proviene de colectas de exploración biológica llevadas a cabo en el Noroeste de Perú, departamento de

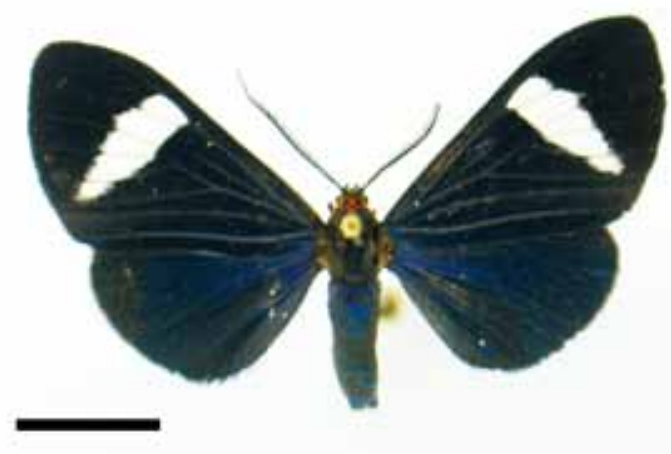

Figura 4. Ctenucha tapajoza Dognin, 1923. Macho. Escala $1 \mathrm{~cm}$.

Piura. Este el registro es el más meridional de la especie.

Material examinado: PIURA: 1 ô, Canchaque, $05^{\circ} 22^{\prime} \mathrm{S} /$ 79³6’W, 1200m, 29.vi.2005 (J. Grados).

Otras especies del género registradas para Perú son:

gynamorpha Hampson, 1898 (Aclytia)(MUSM)

heber (Cramer. 1780) (Sphinx) (MUSM)

hoffmannsi Rothschild, 1912 (Aclytia) (MUSM)

klagesi Rothschild, 1912 (Aclytia) (MUSM)

petra Schaus, 1892 (Aclytia)(USNM)

pydna Druce, 1899 (Aclytia) (MUSM)

reducta Rothschild, 1912 (Aclytia) (MUSM)

punctata Butler, 1876 (Aclytia)(MUSM)

\section{Ctenucha tapajoza Dognin, 1923}

(Fig. 4)

El género Ctenucha Kirby consta de más de 30 especies, con varias de ellas circunscritas por encima de los $3000 \mathrm{~m}$ en la Cordillera de los Andes. La especie Ctenucha tapajoza fue descrita de dos ejemplares machos capturados por el célebre colector alemán Anton Heinrich Fassl (1876-1922) del Río Tapajoz, Brasil.

El nuevo registro proviene de colectas llevadas a cabo en la Cordillera Sierra del Divisor, frontera entre Brasil y Perú.

Material examinado: LORETO: $2 \hat{\jmath} \widehat{\jmath}$, Zona Reservada Sierra del Divisor, Qda. Nubuya, 06 59 '46.55"S / 7358'43.9”W, 205m, 5-8.iii.2009 (C. Espinoza); 2 ôึิ, Zona Reservada Sierra del Divisor, $12.5 \mathrm{~km}$ E Puerto Venus, Río Tapiche, 065'21.66”S / 7401'27.85”W, 225m (C. Espinoza).

Otras especies del género registradas para Perú son:

albipars Hampson, 1901 (Ctenucha) (MUSM)

aymara (Schaus, 1892) (Gangamela)(Perú)*

biformis Dognin, 1907 (Ctenucha)(USNM)

cajonata Dognin, 1923 (Ctenucha)(USNM)

cyaniris Hampson, 1898 (Ctenucha) (MUSM)

garleppi Rothschild, 1912 (Ctenucha)(Cusco)* 


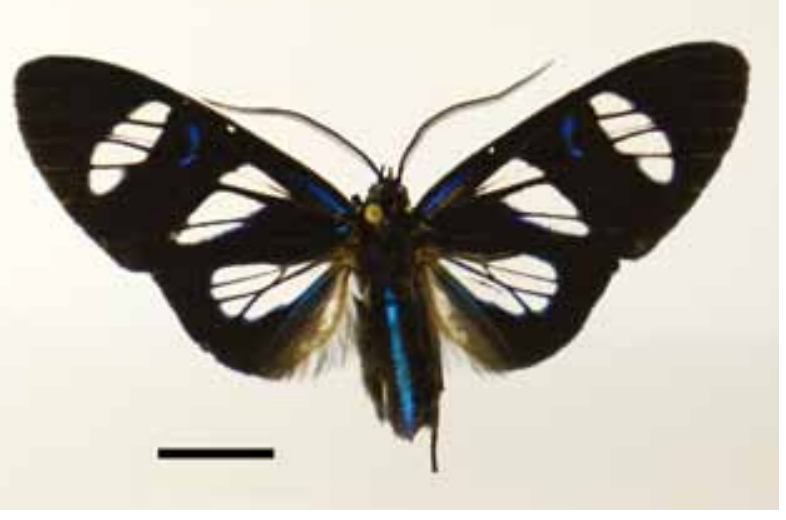

Figura 5. Agyrtiola niepelti Gaede, 1926. Macho. Escala $1 \mathrm{~cm}$.

mortia Schaus, 1901 (Ctenucha)(AMNH)

nantana (Walker, 1864) (Ammalo) (MUSM)

projecta Dognin, 1904 (Ctenucha) (MUSM)

reducta Rothschild, 1912 (Ctenucha)(Oconeque, Agualani, Limbani, Puno)*

refulgens Dognin, 1899 (Ctenucha) (MUSM)

rubrovenata Rothschild, 1912 (Ctenucha) (MUSM)

venosa Walker, 1854 (Ctenucha) (MUSM)

SUBTRIBU: EUCHROMIINA

\section{Agyrtiola niepelti Gaede, 1926}

(Fig.5)

Agyrtidia olivensis Machado \& Barros, 1970. Nuevo sinónimo.

El género es monotípico y la especie fue descrita de un macho capturado en São Paulo de Olivença (Brasil) por Herrn Niepelti, a quien fue dedicada la especie. La especie es algo rara, siendo alopátricas con Agyrtidia uranophila (Walker, 1866), con la que es muy parecida en el patrón de coloración alar. No se conoce las hembras de esta especie, pero es posible que haya pasado desapercibida con hembras de $A$. uranophila (Walker).

Planteamos a Agyrtidia olivensis como nuevo sinónimo de Agyrtiola niepelti. Los holotipos de ambas provienen de la misma

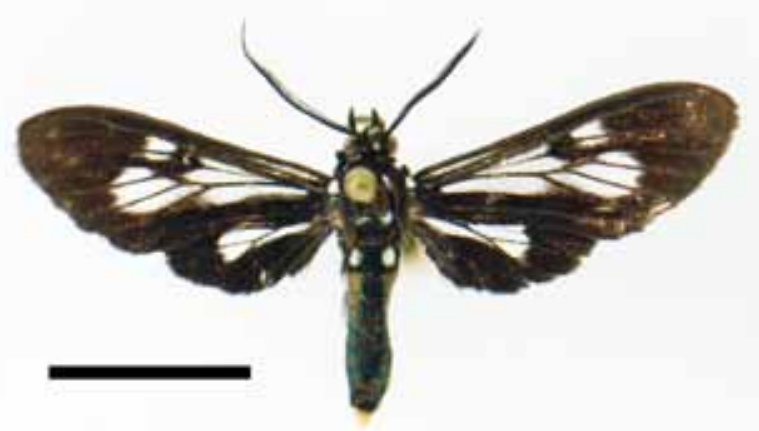

Figura 7. Mystrocneme atavia Hampson, 1898. Macho. Escala $1 \mathrm{~cm}$.

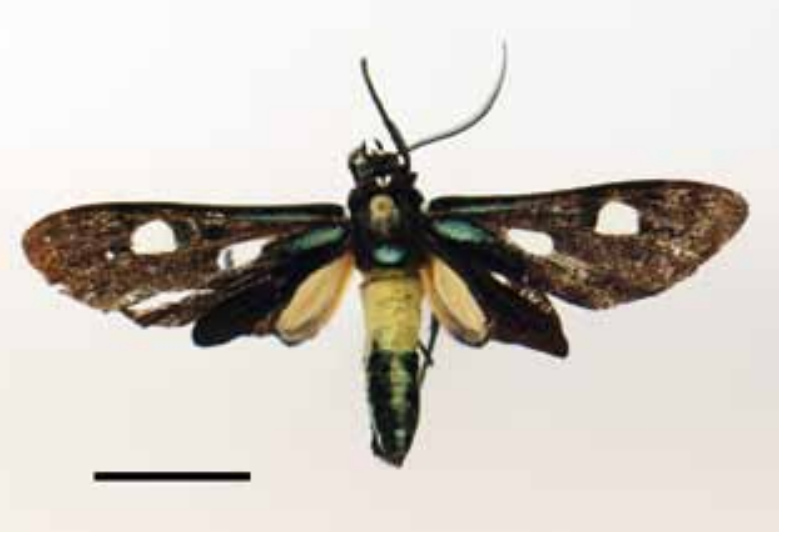

Figura 6. Metaloba argante Druce, 1897. Macho. Escala $1 \mathrm{~cm}$.

localidad, São Paulo de Olivença (Brasil) y porque al analizar ambas especies en su morfología externa, presentan los mismos caracteres, incluyendo las patas posteriores ensanchadas con abundante vellosidad.

Se diferencia de Agyrtidia uranophila (Walker) porque presenta penacho de pelos en la parte interna de las alas posteriores y presencia en el fémur, tibia y el primer segmento del tarso de las patas posteriores de abundante vellosidad. Las diferencias a nivel de genitalia las encontramos en Machado \& Barros (1970).

Material examinado: LORETO: 1 $\widehat{\jmath}$, San Juan de Poli, 03³7'S / 73²4'W, 120m, 19.x.2010 (J.J. Ramírez)(Colectado de día con red).

\section{Metaloba argante Druce, 1897}

(Fig. 6)

El género consta de sólo dos especies, habiendo sido descrita la especie $M$. argante Druce, 1897 de Sarayacu, Ecuador, de un ejemplar macho y una hembra colectados por Clarence Buckley, que colectó varios grupos de fauna en Ecuador y Bolivia. Buckley colectó varias especies de lepidópteros, algunas consideradas raras, ayudado en sus viajes por aquel país por el ecuatoriano Manuel Villagomes (Sclater y Salvin 1876, Hewitson 1870, Vane-Wright 1991). Algunas especies fueron descritas por Herbert Druce (1846-1913). Los ejemplares están actualmente en la colección del Museo Británico (BMNH).

$\mathrm{Al}$ parecer la especie es poco común, conociéndose pocos ejemplares en colecciones en todo el mundo. El nuevo reporte proviene del departamento de Madre de Dios, en el Sudeste de Perú.

Material examinado: MADRE DE DIOS: $1 \hat{\jmath}$, CICRA, Río los Amigos, 12³3'36.3”S / 7006'17.3”W, 380m, 23.xi.2005 (P. Centeno); 1 ऽ, mismos datos que el anterior excepto 04.ix.2005.

\section{Mystrocneme atavia Hampson, 1898}

(Fig. 7)

La especie fue descrita de un ejemplar macho y una hembra proveniente de Fonte Boa, Río Amazonas (Brasil).

El nuevo reporte proviene de colectas realizadas en el este de 
Perú, cerca con la frontera con Brasil. Esta es la segunda especie que se registra para el país.

Material examinado: LORETO: $1 \hat{\jmath}, 37 \mathrm{~km}$ NE Monte Alegre, Río Tapiche, 06²3’01"S / 7404’35”W, 175m, 18-19.x.2008 (A. García): 4 ô̄ Yaquirana, 06 ${ }^{\circ} 14^{\prime} 50^{\prime \prime} \mathrm{S} / 7^{\circ} 17^{\prime} 32^{\prime \prime W}, 165 \mathrm{~m}, 04-06 . x .2008$ (A. García).

La otra especie que ha sido registrada para el Perú es:

varipes (Walker, 1854) (Euchromia) (MUSM)

\section{Agradecimientos}

Deseamos agradecer a Patrick Blandin por la ayuda en visitar la colección del Muséum National d'Histoire Naturelle, Paris (MHNP) y a Joel Minet por la facilidades brindadas en la accesibilidad a la colección de Arctiini (Lepidoptera) formadas por Hervé de Toulgoët. Lo propio conWolfram Mey por la accesibilidad a la colección del Zoologisches Museum, Humboldt Universität, Berlín (ZMHB). El Ministerio de Agricultura proporcionó los respectivos permisos de colecta. Los trabajos dentro de la Zona Reservada Sierra del Divisor fueron realizados con la autorización N038 C/C-2008-INRENA-IANP.

\section{Literatura citada}

Arona de, J. [1891] 1971. La Inmigración en el Perú. Academia diplomática del Perú. 261 pp.

Bachmann C.J. 1918. De Lima al Madre de Dios. Apuntes de viaje. Oficina Tipográfica "La Opinión Nacional", Lima-Perú. 189 pp.

Bailey S.I. 1906. A new peruvian route to the plain of the Amazon. Natn. geogr. Mag. 15:432-447.

Cerda J. 2008. Euchromiini de Guyane Française: (Lepidoptera: Arctiidae, Arctiinae), 171 pp. +2 CDs. Publicado por el autor, Los Angeles: Distributed by Entomological Reprint Specialists.

Druce H. 1884. Insecta. Lepidoptera-Heterocera. Biologia cent.-am. Vol. 1, $\mathrm{xxxi}+490 \mathrm{pp}$.

Druce H. 1906. Descriptions of some new species of Heterocera from Tropical South America. Ann. Mag. Nat. Hist. (7) 18:77-94.
Grados J. 2002. Notas sinonímicas sobre Ctenuchini y Euchromiini (Lepidoptera: Arctiidae). Rev. per. Biol. 10(1):98 - 100.

Hampson G.F. 1898. Catalogue of the Lepidoptera Phalaenae in the British Museum 1. xxi+559 pp., London.

Hampson G.F. 1901. Catalogue of the Arctiidae and Agaristidae in the collection of the British Museum 2. xx+690 pp., London.

Harter, E. 1907. George Richard Ockenden. Novit. zool. 14(1):341-342.

Heppner H.B. \& G. Lamas. 1982. Acronyms for World Museum Collection of Insects, with an Emphasis on Neotropical Lepidoptera. Bull. ent. Soc. Am. 28(3):305-315.

Hewitson W.C. 1870. Descriptions of twenty-two new species of Equatorial, Lepidoptera. Trans. R. ent. Soc. Lond. 18(2):153-163.

Jordan K. 1938. In memory of Lord Rothschild, Ph.D., F.R.S., J.P. born the 8th February, 1868, died the 27 $7^{\text {th }}$ August, 1937. Novit. zool. 41(1):1-41.

Lamas G. \& J. Grados. 1996. Sinopsis de los Pericopinae (Lepidoptera: Arctiidae) del Perú, con comentarios taxonómicos y la descripción de una nueva subespecie. Rev. per. Ent. 39:21-28.

Lamas G. 1980. Introducción a la historia de la entomología en el Perú. Rev. per. Ent. 23(1):17-37.

Machado J. \& A.R. Barros. 1970. Ctenuchidae Neotropicais V. Redesdcriāo do gênero Agyrtidia Hampson, 1898 e descrição de nova especie (Lepidoptera-Heterocera). Bolm. Mus. nac. Rio de J. Nova Serie, Zoologia. 278:17 pp.

Rothschild 1912. New Syntomidae. Novit. Zool. 19:151-186.

Rothschild W. 1909. Descriptions of some new South American Arctiadae, with notes. Novit. Zool. 16(1):21-52.

Rothschild W. 1910. Catalogue of the Arctianae in the Tring Museum, with notes and descriptions of new species. Novit. Zool. 17(1):1-85.

Scatler P.L. \& M.A. Salvin. 1876. Descriptions of new birds obtained by Mr. C. Buckley in Bolivia. Proc. zool. Soc. Lond. 44:253-254.

Schaus, W. 1892. Descriptions of new species of Lepidoptera Heterocera from Brazil, Mexico and Peru. Part. I. Proc. zool. Soc. Lond. 272-291.

Sobrevilla N. 2001. La creación de la colonia de Pozuzo. pp. 167-230. En: La presencia europea en el Perú. G. Bonfiglio. Fondo editorial Congreso del Perú, 381 pp.

Vane-Wright R.I. 1991. A Portrait of Clarence Buckley, Zoologist. The Linnean 7(3):30-33.

Watson A. \& D. Goodger. 1986. Catalogue of the Neotropical Tiger-moths. Ocassional Papers on Systematic Entomology 1.71 pp.

Zerny, H. 1912. Syntomidae. En Lepidopterum Catalogus, Pars 7, H. Wagner editor, W. Junk, Berlin. 179 pp. 
